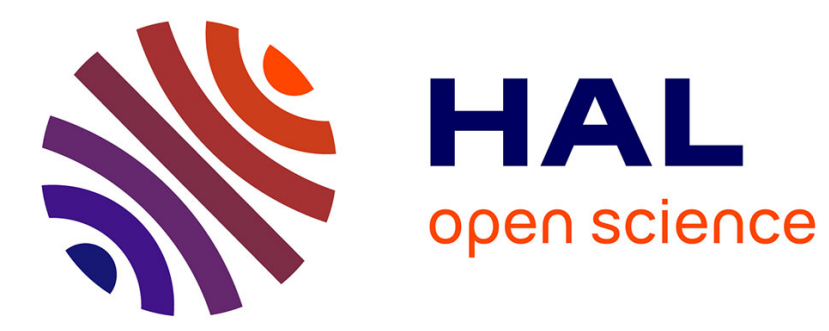

\title{
Improving Decision-Making for Fuzzy Logic-based Routing in Wireless Sensor Networks
}

\author{
Ehsan Ahvar, Antonio M Ortiz, Noel Crespi
}

\section{To cite this version:}

Ehsan Ahvar, Antonio M Ortiz, Noel Crespi. Improving Decision-Making for Fuzzy Logic-based Routing in Wireless Sensor Networks. 2013 IEEE 10th International Conference on Ubiquitous Intelligence \& Computing (UIC Symposium), Dec 2013, Vietri sul Mere, Italy. hal-01111504

\section{HAL Id: hal-01111504 \\ https://hal.science/hal-01111504}

Submitted on 30 Jan 2015

HAL is a multi-disciplinary open access archive for the deposit and dissemination of scientific research documents, whether they are published or not. The documents may come from teaching and research institutions in France or abroad, or from public or private research centers.
L'archive ouverte pluridisciplinaire HAL, est destinée au dépôt et à la diffusion de documents scientifiques de niveau recherche, publiés ou non, émanant des établissements d'enseignement et de recherche français ou étrangers, des laboratoires publics ou privés. 


\title{
Improving Decision-Making for Fuzzy Logic-based Routing in Wireless Sensor Networks
}

\author{
Ehsan Ahvar \\ Institut Mines-Telecom, \\ Telecom SudParis, France \\ Antonio M. Ortiz \\ Institut Mines-Telecom, \\ Telecom SudParis, France \\ Institut Mines-Telecom, \\ Telecom SudParis, France \\ Email: ehsan.ahvar@telecom-sudparis.eu Email: antonio.ortiz_torres@it-sudparis.eu Email: noel.crespi@mines-telecom.fr
}

\begin{abstract}
The task of routing data from a source node to the base station is a critical issue in Wireless Sensor Networks (WSNs). Fuzzy logic is the main proposal of a number of papers in the literature as an effective method for making decisions to transfer data towards the destination. Although fuzzy logic has a very important role in designing routing protocols for WSNs, identifying its fuzzy sets and defining best possible rules is a complex challenge. This paper introduces Improved-fuzzy logic (I-fuzzy), a simple and effective method that helps to address the weakness of fuzzy logic in terms of defining rules. The Ifuzzy method is tested in several scenarios by using GloMosim simulator and compared to a classic fuzzy logic approach and to a traditional minimum hop routing. The results show that the I-Fuzzy method outperforms the other approaches in terms of data delivery, energy conservation and load distribution.
\end{abstract}

\section{INTRODUCTION}

Wireless Sensor Networks (WSN) are usually comprised of battery-operated sensor devices capable of communication and processing [3]. This type of networks can be used in a number of applications to monitor and analyze complex phenomena over a large region and for a long period of time [12]. The nodes that compose WSNs can collect physical values such as humidity, temperature, pressure, lighting, oxygen level, presence, etc. Their ability to obtain these values combined with the possibility of autonomous operation and wireless communication makes these networks particularly appealing in applications such as environmental monitoring, smart cities, home automation, smart agriculture, industrial control, health care, logistics, security, etc.

Node deployment, data collection and analysis are the three main stages that define the operation of a WSN. Issues such as node redundancy, node failure, path break or data loss must be addressed to ensure satisfactory performance. One of the most critical aspects that directly impacts the overall performance of WSNs is routing.

The task of routing data from a source to a destination is an important procedure for any network. An efficient routing protocol, in conjunction with effective medium access control, allows for correct operation of the network by ensuring that data will be delivered to the destination. Several objectives can be targeted to evaluate the efficiency of a routing proposal, e.g., end-to-end delay, energy efficiency and load balance, depending on the type of network. In WSNs, where nodes are constrained in terms of computing capabilities, memory, storage, and specially energy, an efficient routing approach contributes to balancing the network load, with the ultimate objectives of ensuring data delivery and extending the network's lifetime.

Several methods can be used to improve routing efficiency in WSNs, one such method is the creation of clusters i.e., groups of nodes commanded by one central node [1], to reduce the number of transmissions. The consideration of several variables such as the number of hops, residual node energy levels, channel state, etc., is the main contribution of a number of proposals. The parameter that is used by many of these approaches is the number of hops [4], [17], but considering multiple parameters usually offers even better performance [2]. One method that has shown good results uses fuzzy logic to combine multiple parameters [15], [20].

Fuzzy Logic [19] is a decision system approach that works similarly to human control logic. It is a useful technique since it uses human language to describe inputs and outputs, and provides a simple method for reaching a conclusion from imprecise, vague or ambiguous input information. Fuzzy-logic systems include a fuzzy-rule set to define the relationship between the input and output variables. Furthermore, just a few data samples can provide quite accurate results. The potential of fuzzy logic is being fully explored in a range of fields such as signal processing, speech recognition, aerospace, robotics, embedded controllers, networking and marketing [9].

This paper focuses on improving decision making in fuzzy logic for routing in WSNs. The main contribution of this paper is the proposal of an assistant module (AM) for fuzzy logic controllers in WSN routing. The AM is a simple and effective technique that computes an independent metric considering the same parameters as fuzzy logic. This method can significantly improve the performance obtained with traditional fuzzy logicbased decision making. Furthermore, a Decision Module is also proposed in order to select the best routes in WSN communications.

The rest of this paper is organized as follows. Section II depicts the related work. A general view regarding fuzzy logicbased routing is shown in Sec. III. The main contribution of this paper, the assistant module for fuzzy logic-based routing in WSNs is presented in Sec. IV] The experimental evaluation is detailed in Sec. $\mathrm{V}$, where the proposed approach is compared to traditional fuzzy logic and minimum hop routing. Finally, Sec. VI presents the conclusions and exposes the future work. 


\section{RELATED WORK}

There are a number of papers that use fuzzy logic to improve the performance of routing protocols for WSNs. The work in [6] presents a fuzzy link quality estimator that makes use of fuzzy logic to combine four link-quality properties: channel quality, stability, asymmetry and packet delivery. The evaluation of this proposal is focused on one-hop networks, and no information about multihop networks is given. The use of fuzzy logic to improve the LEACH protocol is proposed in [18]. Combining node density, residual energy and distance using fuzzy logic, the obtained results show that LEACH-FL improves decision making for cluster head selection. Experiments were carried out in a 20-node network; experiments with a higher number of nodes would be desirable to evaluate the scalability of their proposal.

Fuzzy logic has also been used to improve the efficiency of the Ad-hoc On-demand Distance Vector (AODV) protocol. The AODV-FL is presented in [16], where fuzzy logic is used to evaluate nodes and limit the scope of message flooding, thereby efficiently reducing the network traffic and enabling the network to extend its lifetime. Minor changes in the network discovery procedure were also proposed with the aim of improving the performance of the route creation process.

Haider et al. propose in [11] a method based on fuzzy logic to optimize cluster-based routing protocols. They accurately define the operation of fuzzy logic-based systems for routing enhancement, but they neglect important parameters such as route depth and communication time in the experiments. These works reinforce using fuzzy logic as a useful technique to improve the performance of routing protocols.

The performance of fuzzy logic techniques can also be improved, with the aim of outperforming their initial advantages, thus enhancing even more the results obtained when they are employed. Two examples of fuzzy logic improvement techniques can be found in [5] and [13].

Usually, the fuzzy rule set is defined by an expert in the field of interest who can link the facts with the conclusions. This procedure sometimes fails to obtain an optimal behavior when dealing with nontrivial problems. The work presented in $[5]$ is focused on improving rule selection in fuzzy logic systems. They propose the use of two advanced tuning techniques (lateral and LA-tuning) combined with rule selection to improve on the fuzzy logic-based controllers obtained by experts in nontrivial problems. These tuning techniques are focused on the variation of the shape of the membership functions, and they improve global interaction by inducing better cooperation among the rules.

Genetic algorithms [10] can also be used for the optimization of decision making techniques such as fuzzy logic. In [13], a fuzzy logic controller is proposed to improve the maximum power point tracking in photovoltaic systems. This fuzzy controller is then assisted by genetic algorithms that obtain the best subsets of the membership functions with the aim of improving the performance of the fuzzy controller.

These last two techniques improve the membership functions in fuzzy logic-based controllers. Our proposed approach aims at enhancing fuzzy logic decision making by reevaluating fuzzy parameters in order to balance them, thereby increasing the efficiency of the routing mechanism.

\section{FUZZY LOGIC-BASED ROUTING FOR WSNS}

This section gives a brief introduction related to the general operating mechanism of fuzzy-based routing protocols.

\section{A. General Mechanism}

The operation of fuzzy logic-based protocols can be generally divided into two steps:

Network setup: Once the network has been deployed in the area where it is to operate, the base station transmits a broadcast packet. When a node receives this initial message, it checks whether it has an entry in its neighbor table for the node that transmitted the message. If not, the receiver node adds an entry that consists of the information received (i.e., neighbor address, hop count and energy level). The node then increments the hop count stored in the message and sets this hop count as its own hop count. It then retransmits the broadcast, but changes the message information to include its own (i.e., local node address, number of hops and residual energy level). Normally every node in the network retransmits the broadcast message once to all of its neighbors, but for accuracy, in case of a node receiving a broadcast message with a lower hop count than the stored one, it updates its hop count and broadcast the massage again. When this initial broadcast has been flooded through the network, each node knows its hop count and has the address, hop count and residual energy level of each of its neighbors. In this situation, every node in the network has now enough information to send and route messages towards the base station. The routes to be selected will depend on the metric used in each case (e.g., shortest path, fuzzy logic metric, etc.).

Data transfer: When a node observes an event, it should initiate a routing process to send data packets towards the base station. Traditional fuzzy logic-based routing protocols consider several metrics (i.e., hop count, energy level) and merge all these metrics into one single metric that is used to select the next hop. Data is then sent to the next hop until reaching the base station. The execution of these kind of mechanisms does not affect the overall network performance (in terms of processing time), what is more, it usually increases the network performance thanks to the load balance that is done when selecting the node with best state as next hop.

\section{B. Fuzzy Logic Module}

In order to improve routing performance in WSNs, fuzzy logic can be used as a metric that allows the combination of several parameters into one single metric. In this work, a traditional fuzzy logic system is used to estimate the best node to send data towards the base station. Later, in Sec. IV, a method to improve fuzzy logic performance is proposed.

The fuzzy logic module has the ability of combining several metrics into one single metric. For that, four stages are needed: fuzzification, rule evaluation, combination or aggregation of rules, and deffuzification (see Fig. 1). The input of the fuzzy logic module is usually a crisp value. To allow this value to be processed by the system, it has to be converted to natural language, that is, it has to be fuzzified. In this way, the fuzzifier 


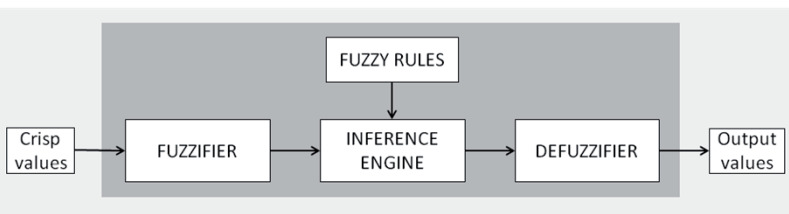

Fig. 1. Fuzzy logic module operation.

method takes numeric values and turns them into fuzzy values which can be processed by the inference system. These fuzzy values represent the membership values of the input variables to the fuzzy sets.

Once values have been fuzzified, the inference system processes the fuzzy rules to get a fuzzy output. In the case of a fuzzy rule having more than one antecedent (conditional element), an AND (minimum) or OR (maximum) operator is used to estimate the output value of rule evaluation.

The third step is the aggregation of all outputs, where the outputs of each rule are combined to form a new fuzzy set.

Finally, at the deffuzification stage, the new aggregated fuzzy set is converted to a number. Our fuzzy logic module uses the centroid technique which determines the point where a vertical line divides the combined set into two equal parts. This deffuzification method is sensitive to all the rules [8] and it is easy to compute in the resource constrained WSN nodes.

The parameters considered in this work, that will be combined through fuzzy logic are: number of hops to the base station and residual energy level. The number of hops to the base station represents the number of times that a message must be forwarded to reach the base station and be further analized. The residual energy level in the nodes is a critical parameter in WSNs, where nodes are usually battery powered, and efficient energy saving and balance leads to an extension of the overall network lifetime. These parameters are used as an example to show the efficiency of the proposed technique. Other parameters such as channel properties or network congestion can be also used, just adapting fuzzy sets and rules.

The membership functions for the input and output parameters used in our fuzzy logic model are detailed in Fig. 2. Note that both parameters (number of hops and residual energy level) are represented with equivalent membership functions. This simplifies operations in the fuzzy logic engine and increases its efficiency when executed in the WSN nodes.

For example, considering the number of hops, labell corresponds to low, label2 to medium and label3 to high. The values $X 0$ to $X 4$ have been adjusted according to each input variable. Continuing with the example of residual energy, $X 0=0, X 1=25, X 2=50, X 3=75$, and $X 4=100 \%$, considering $100 \%$ when node batteries are fully charged. Also, input parameters are characterized into a set of linguistic values: Number of hops [low, medium, high], Energy Level [low, medium, high], and Output [low(bad), medium, good (high)]. Linguistic input values are related to output values following a set of rules that is defined by an expertise in the field [14]. The FL system used in this papers follows the rules defined in Tab. I. that represents rules such as:

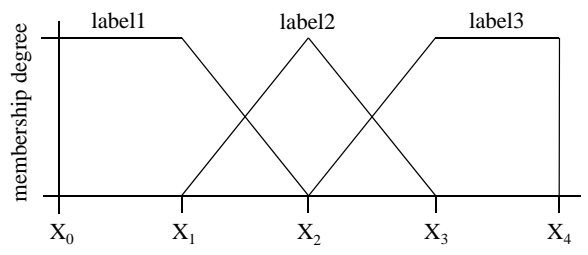

Fig. 2. Membership function.

TABLE I. FUZZY RULE BASE

\begin{tabular}{lll}
\hline Energy level & Number of hops & Output \\
\hline Low & Low & Medium \\
Low & Medium & Low \\
Low & High & Low \\
Medium & Low & Medium \\
Medium & Medium & Medium \\
Medium & High & Low \\
High & Low & High \\
High & Medium & Medium \\
High & High & Low \\
\hline
\end{tabular}

IF Number of hops is high AND energy level is low THEN Output is good (high). When new values come to the fuzzy logic model, they are fuzzified to linguistic values, and correspondent rules are triggered. To calculate the final result, the output values are deffuzified to provide a numerical value that will be used as a metric during the routing process. According to our rule definition, the higher the value, the more appropriate the node is to be selected as next hop. In some occasions, fuzzy logic by itself does not provide the best result. This can happen due to several reasons:

- Membership functions do not represent all the possible situations, specially in threshold values between two consecutive fuzzy sets.

- Rules defined by the expert do not adjust to the desired behavior, and produce output values that are not optimal. This is very common, since it is very difficult to know a priori the results that will be produced by the system when combining several rules.

- There is noisy input values that will produce an undesired output value.

To mitigate as much as possible these situations and with the aim of improving the FL system performance, next section presents the main contribution of this paper. An assistant module that is executed in parallel to the FL system. This assistant module computes an independent metric by considering the same parameters as fuzzy logic. In order to break possible ties and to decide the final output, a decision maker is used to decide which output value will be considered: the one provided by fuzzy logic, or the output of the assistant module.

\section{Assistant Module for Fuzzy Lo-gic BASED ROUTING}

In this section, we introduce an Assistant Module (AM), a simple but effective technique that can significantly improve the performance obtained with traditional fuzzy logic methods.

When a node $i$ has a packet to send, or it receives a packet to forward, the routing process is invoked. The Fuzzy Logic 
(FL) selects the ID of the next-hop, $I D^{(F L)}$, based on the values stored in the neighbor table. In parallel, the $A M$ with access to the neighbor table also selects the next-hop based on an alternative criterion, which can be denoted as $I D^{(A M)}$. A Decision Maker module (DM) then selects the next-hop that will be finally used.

The operation of the AM can be summarized as follows: when a node $i$ has a packet to send or receives a packet to forward, the routing process begins by the AM computing a weight for each neighbor (i.e, neighbor $j$ ) based on the cost function described in (1).

$$
W_{i j}(t)= \begin{cases}\omega \mathcal{E}_{j}(t)\left(1-\frac{H_{j}(t)}{H^{\max }}\right) & \frac{\mathcal{E}_{j}(t)}{<\text { Threshold }>}>1 \\ 0 & \frac{\mathcal{E}_{j}(t)}{<\text { Threshold }>} \leq 1\end{cases}
$$

where $\mathcal{E}_{j}(t)$ is the energy level of neighbor $j, \omega$ is a control parameter that simply limits the energy factor $\omega \mathcal{E}_{j}(t)$ to the interval $[0,1] \forall j$, and $H^{\max }$ is the maximum number of hops reported among all the neighbors of node $i$. Note that the energy threshold in (1) is used to remove from the selection process those neighbors $j$ whose energy level is very low. The rationale of using (1) is that, it produces a good balance between energy and the hop count in the form of a simple compound metric. Next, the AM selects the neighbor with highest evaluation value as a next hop and sends its ID to the decision maker.

The processing of the Decision Maker module is summarized in Alg. 1. If the selections made by the FL and AM modules matches, then the selected node will become the next-hop and data will be sent to it. Otherwise, the DM runs a basic sequence of tie-breaking rules until the next-hop is selected. The main contribution of this paper is to show how this combined selection outperforms any possible selection of the isolated fuzzy logic.

The use of the AM in conjunction with traditional fuzzy logic is called Improved-Fuzzy (I-Fuzzy). A performance evaluation of the proposed mechanism and comparison with

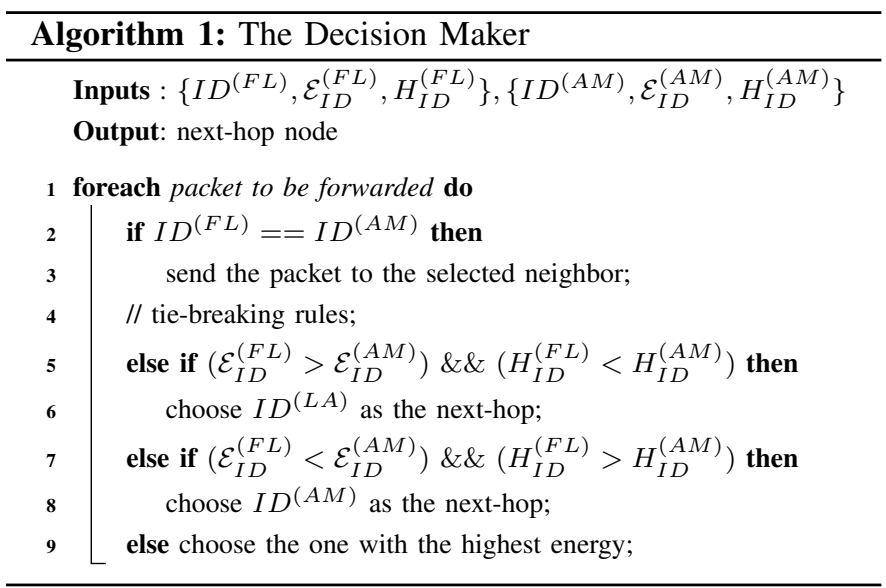

the traditional fuzzy logic and minimum hop count methods is presented.

\section{Evaluation}

This section presents a performance evaluation of the Improved-Fuzzy mechanism by comparing it to Fuzzy-based routing, and to Minimum Hop Routing (MHR) [7] as a basic routing protocol. The GloMoSim simulator developed by UCLA [21] was used to realize the simulations. The simulation model and the results obtained are described below.

\section{A. Simulation Model}

The simulation surface considered was $500 \mathrm{~m} \times 500 \mathrm{~m}$. The radio range was set to $50 \mathrm{~m}$, with an available bandwidth of $2 \mathrm{Mbps}$, a radio transmission(TX) power of $-7.0 \mathrm{dBm}$, and packet size is 512 bits. Each simulation had a 150-minute duration, and the tests were run under various conditions, such as with different numbers of nodes, namely, 800, 900, and 1000 nodes, and with 10 different seeds. During simulation, a sensor is randomly selected every 10 seconds, which initiates a packet transmission to one of its neighbors towards the base station $S$. Moreover, node placements in the terrain were selected randomly. It is worth mentioning that, even though the placement and initial energy of the nodes were set randomly, once those factors were set, they remained fixed for rest of the trials to obtain comparable results across experiments.

The simulation model comprises two different scenarios:

Scenario I-In this scenario, we assume a critical situation, where the energy levels for transmission mode are very low (i.e. $0.00005 \mathrm{~W}$ ). Under these conditions, we evaluate the different routing schemes considering three different tests:

Test 1: Time until the first path is broken-When a node wants to send/forward a data packet but no alive neighbors are available, it leads to a path break. This test is one of the indicators of the effectiveness of routing schemes in terms of energy management and traffic balance.

Test 2: Number of broken paths-This test computes the total number of paths that fail for each routing scheme during a simulation period of 150 minutes.

Test 3: Number of node failures at the end of the simulation-This test computes the total number of nodes that fail for each routing scheme during a simulation period of 150 minutes.

Test 4: Percentage of packet deliveries at the end of the simulation - The efficiency of packet delivery is another important factor for determining protocol quality. Scenario II-In this scenario, the nodes' energy level is set sufficiently high so as to avoid experiencing node failures during the simulation runtime. Our goal in this case is to compare the fairness in terms of path distribution and energy consumption. In order to avoid bias in the comparison, we ensure that all the routing schemes transmit the same amount of data, and that this occurs without node failures. We carry out two tests to examine how the routing schemes save and manage energy in regular operation mode.

Test 5: Variance in the remaining energy levels of the neighbors of base station $S$-This test makes it possible to see 


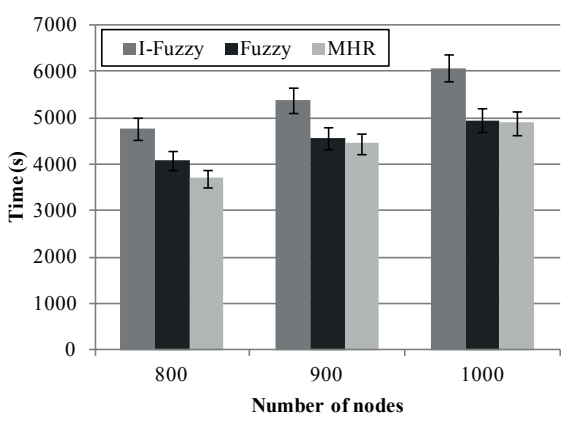

(a) Time until the first path is broken.

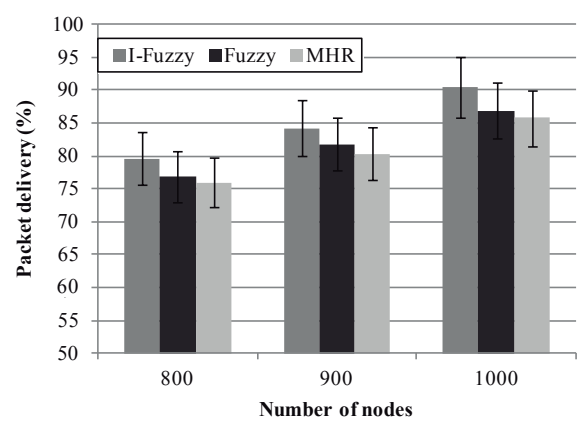

(d) Packet delivery.

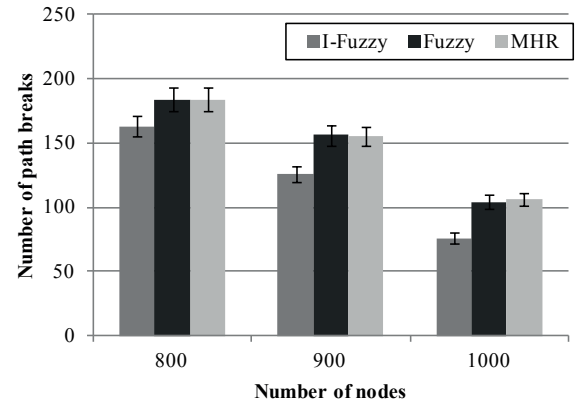

(b) Number of broken paths.

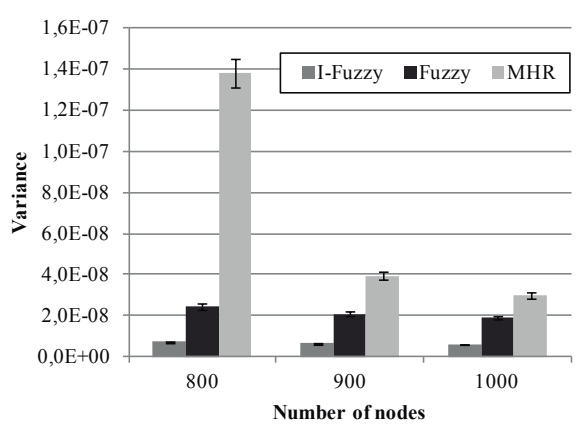

(e) Variance of the remaining energy for the base station neighbor nodes.

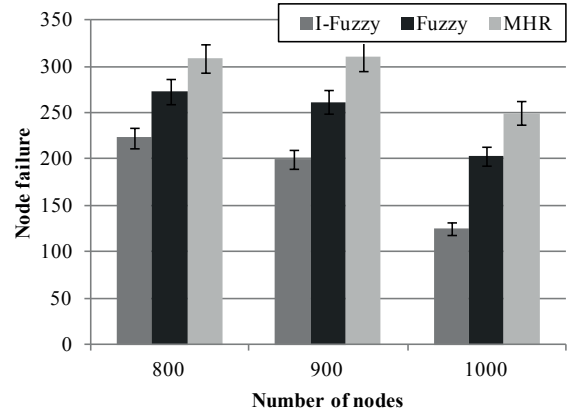

(c) Number of nodes that fail due to energy run out.

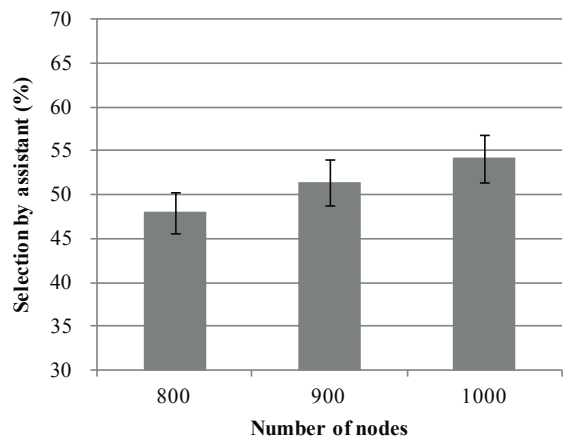

(f) Effect of the Assistant Module in decision making.

Fig. 3. Simulation Results.

which routing scheme is best at performing energy balancing among the nodes closest to the base station.

Test 6: Effect of AM on Fuzzy decisions - To understand the role of the Assistant Module in fuzzy decisions, this test analyzes the impact of AM decisions in the selection of the next hop.

\section{B. Simulation Results}

1) Scenario I: In case of no live neighbor available to be selected as a next hop, a path broke occurs. Fig. 3(a) details the outcome of Test 1 (i.e., the time elapsed until the first path is broken). These results provide a first indication of the performances obtained with the different routing schemes under evaluation, since once that path break happens, new routes must be selected, and data loss occurs. It can be observed that the first path failures are relatively similar for the basic fuzzy and MHR methods. As indicated in Fig. 3(a) when using the Assistant Module, the first path fails only after $\sim 80$ to $\sim 100$ minutes depending on the number of nodes present in the network. These results show that the use of IFuzzy provides an improved load balancing among network nodes, allowing them to use all the possible data paths for longer than is possible with the other approaches.

As time goes by, more paths are broken. Figure 3(b) shows the number of path breaks after 150 minutes for all the approaches. The best performance for this test is achieved by the I-Fuzzy method, outperforming the other approaches thanks to its better network load distribution. It is worth mentioning that as the number of nodes grows, the amount of broken paths decreases, since more nodes are available, and thus more paths can be selected.

During network operation, it is desirable that all nodes or at least as many as possible, are available to be selected as next hops and thus ensuring correct packet routing. When nodes run out of energy, they start to fail, and the overall network performance is at risk. Fig. 3(c) shows the number of node failures observed in the experiments. It can be observed that the number of nodes that fail for the I-Fuzzy scheme much less than in the other approaches. Just as with the number of broken paths, as the network size increases, more nodes are available to be selected as next hops, providing for better load balancing and enabling nodes to stay alive longer.

Fig. 3(d) shows the percentage of successful packet deliveries after 150 minutes for all the approaches. Again, the best results for this test are for the I-Fuzzy approach, thanks to the load balance and better decision making performed by this technique. It is worth mentioning that as the number of network nodes grows, packet delivery increases for all the approaches, due to the higher number of available paths in the network.

2) Scenario II: This second scenario is designed to evaluate the load distribution and fairness in terms of packet transfer. As mentioned earlier, in this case the batteries are not in a critical state, and thus, we can examine the regular behavior of the different routing schemes normal situations. 
How the load is distributed among network nodes will directly impact the overall network performance, as will the amount of energy available in the nodes. The nodes located close to the base station (i.e., the base station neighbors) are critical, since all the information coming from the rest of the network will have to be forwarded by them in order to reach the base station. How the energy is distributed among these nodes is an indicator of the likely node and path failures, and the consequent risk of network disconnection and data loss.

The variance of the remaining energy levels of the base station neighbor nodes is shown in Fig. 3(e) The lower the variance is, the better the load has been distributed, and the better the overall performance. I-Fuzzy and simple Fuzzy approaches achieve better results compared to the MHR method, indicating that a fairer distribution of energy consumption has been achieved. The use of MHR ensures shorter routes, but does not consider load balance, and so nodes in the most-used routes are quickly exhausted causing path break and data loss faster than in the other approaches.

To conclude, Fig. 3(f) shows the breakdown of the decisions made by the Decision Maker, indicating the fractions when the selection coincides with the one made by Assistant Module (AM) as well as the fraction where both methods (fuzzy logic and AM) agree in their next hop selection. The results exposed in Fig. 3(f) show that the AM has an important role in making decision and improving the quality of fuzzy logic decisions as it corrected close to 50 percent of the fuzzy logic decisions.

\section{CONCLUSIONS}

Routing is one of the most critical stages in WSNs operation. The correct selection of the next hop directly affects the overall network performance in terms of network congestion, end-to-end delay and energy consumption.

Several techniques have been proposed in the literature to increase the efficiency of routing in WSNs, but most of them have something in common: consider one or several parameters to evaluate the current state of network nodes as a base to make decisions.

Fuzzy logic is a decision making technique that is able to combine several parameters into one single metric and that has shown good performance for the improvement of routing performance in WSNs. Despite of these improvements,some issues can arise, and fuzzy logic can be improved upon. This paper has proposed a simple and effective solution that can work in combination with fuzzy logic, called the ImprovedFuzzy method. This solution is composed of an Assistant Module that computes an independent metric by considering the same parameters as fuzzy logic. A Decision Module has been also proposed to decide which output value will be selected: the one provided by the fuzzy logic module, or the output of the Assistant Module, the I-Fuzzy approach.

The experimental evaluation shows that I-Fuzzy outperforms traditional fuzzy logic decision making. Furthermore, a minimum hop routing approach has also been considered in the experiments in order to show a fair comparison with a classic routing approach.

\section{ACKNOWLEDGEMENTS}

This work was supported by the EU ITEA 2 Project: 11012, "ICARE: Innovative Cloud Architecture for Real Entertainment".

\section{REFERENCES}

[1] A. A. Abbasi and M. Younis. A survey on clustering algorithms for wireless sensor networks. Computer communications, 30(14):28262841, 2007.

[2] K. Akkaya and M. Younis. A survey on routing protocols for wireless sensor networks. Ad hoc networks, 3(3):325-349, 2005.

[3] I. F. Akyildiz, W. Su, Y. Sankarasubramaniam, and E. Cayirci. Wireless sensor networks: a survey. Computer networks, 38(4):393-422, 2002.

[4] J. N. Al-Karaki and A. E. Kamal. Routing techniques in wireless sensor networks: a survey. Wireless Communications, IEEE, 11(6):6-28, 2004.

[5] R. Alcalá, J. Alcalá-Fdez, M. J. Gacto, and F. Herrera. Improving fuzzy logic controllers obtained by experts: a case study in hvac systems. Applied Intelligence, 31(1):15-30, 2009.

[6] N. Baccour, A. Koubaa, H. Youssef, M. ben Jama, D. Rosrio, M. Alves, and L. Becker. F-LQE: A Fuzzy Link Quality Estimator for Wireless Sensor Networks. In Proceedings of the 7th European Conference on Wireless Sensor Networks (EWSN), 2010

[7] S. S. Chiang, C. H. Huang, and K. C. Chang. A minimum hop routing protocol for home security systems using wireless sensor networks. IEEE Transactions on Consumer Electronics, 53(4):1483-1489, 2007.

[8] E. Cox. Fuzzy fundamentals. Spectrum, IEEE, 29(10):58-61, 1992.

[9] S. Ghosh, Q. Razouqi, H. J. Schumacher, and A. Celmins. A survey of recent advances in fuzzy logic in telecommunications networks and new challenges. Fuzzy Systems, IEEE Transactions on, 6(3):443-447, 1998.

[10] D. E. Goldberg. Genetic algorithms in search, optimization, and machine learning. 1989.

[11] T. Haider and M. Yusuf. A fuzzy approach to energy optimized routing for wireless sensor networks. The International Arab Journal of Information Technology, (6):179-188, 2007.

[12] H.-J. Hof. Applications of sensor networks. In Algorithms for Sensor and Ad Hoc Networks, pages 1-20. Springer, 2007.

[13] C. Larbes, S. Ait Cheikh, T. Obeidi, and A. Zerguerras. Genetic algorithms optimized fuzzy logic control for the maximum power point tracking in photovoltaic system. Renewable Energy, 34(10):2093-2100, 2009.

[14] C. C. Lee. Fuzzy logic in control systems: fuzzy logic controller. i. Systems, Man and Cybernetics, IEEE Transactions on, 20(2):404-418, 1990.

[15] Q. Liang and Q. Ren. Energy and mobility aware geographical multipath routing for wireless sensor networks. In Wireless Communications and Networking Conference, 2005 IEEE, volume 3, pages 1867-1871. IEEE, 2005.

[16] A. M. Ortiz, F. Royo, T. Olivares, J. C. Castillo, and L. Orozco-Barbosa. On reactive routing protocols in zigbee wireless sensor networks. Expert Systems: The Journal of Knowledge Engineering, 2013.

[17] C. E. Perkins and E. M. Royer. Ad-hoc on-demand distance vector routing. In Mobile Computing Systems and Applications, 1999. Proceedings. WMCSA'99. Second IEEE Workshop on, pages 90-100. IEEE, 1999.

[18] G. Ran, H. Zhang, and S. Gong. Improving on LEACH Protocol of Wireless Sensor Networks Using Fuzzy Logic. Journal of Information \& Computational Science, (7):767-775, 2010.

[19] L. Reznik. Fuzzy controllers handbook: how to design them, how they work. Newnes, 1997.

[20] T. Srinivasan, R. Chandrasekar, and V. Vijaykumar. A fuzzy, energyefficient scheme for data centric multipath routing in wireless sensor networks. In Wireless and Optical Communications Networks, 2006 IFIP International Conference on, pages 5-pp. IEEE, 2006.

[21] X. Zeng, R. Bagrodia, and M. Gerla. Glomosim: a library for parallel simulation of large-scale wireless networks. In Parallel and Distributed Simulation, 1998. PADS 98. Proceedings. Twelfth Workshop on, pages 154-161. IEEE, 1998 